\title{
Consensus recommendations for the care of children receiving chronic dialysis in association with the COVID-19 epidemic
}

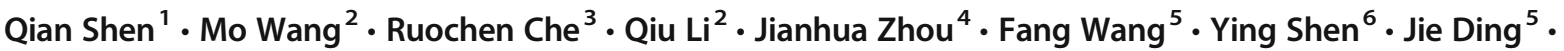 \\ Songming Huang ${ }^{3}$. Hui-Kim Yap ${ }^{7}$ Bradley A Warady ${ }^{8} \cdot$ Hong Xu $^{1}$ - Aihua Zhang ${ }^{3}$. for the Chinese Society of \\ Pediatric Nephrology and Chinese Medical Doctor Association of Pediatric Nephrology
}

Received: 4 March 2020 /Revised: 25 March 2020 / Accepted: 26 March 2020 / Published online: 24 April 2020

(C) IPNA 2020

\begin{abstract}
Coronavirus disease 2019 (COVID-19) has rapidly spread not only in China but throughout the world. Children with kidney failure (chronic kidney disease (CKD) stage 5) are at significant risk for COVID-19. In turn, a set of recommendations for the prevention and control of the severe acute respiratory syndrome coronavirus 2 (SARS-CoV-2) and COVID-19 in pediatric hemodialysis (HD) centers and in home peritoneal dialysis (PD) settings have been proposed. The recommendations are based on the epidemiological features of the SARS-CoV-2 virus and COVID-19 disease, susceptibility factors, and preventive and control strategies. These recommendations will be updated as new information regarding SARS-CoV-2 and COVID-19 becomes available.
\end{abstract}

Keywords COVID-19 · SARS-CoV-2 - Dialysis · Children

Recently, coronavirus disease 2019 (COVID-19), an acute respiratory illness first diagnosed in Wuhan, China, in December 2019 [1, 2], has rapidly spread not only in China but throughout the world. Because of its high infectivity and mortality, the World Health Organization (WHO) has defined COVID-19 as a global pandemic. As of March 23, 2020, the global number of confirmed cases had exceeded 330,000, with more than 14,000 deaths [3]. Over 900 children have been affected, and neonatal infection has also been reported in China [4, 5]. Person-to-person transmission of the virus, severe acute respiratory syndrome coronavirus 2 (SARSCoV-2) as designated by the International Committee on

Qian Shen, Mo Wang and Ruochen Che contributed equally to this work.

Hong Xu

hxu@shmu.edu.cn

Aihua Zhang

zhaihua@njmu.edu.cn

1 Department of Nephrology, Children's Hospital of Fudan University, Shanghai, China

2 Department of Nephrology, Children's Hospital of Chongqing Medical University, Chongqing, China

3 Department of Nephrology, Children's Hospital of Nanjing Medical University, Nanjing, China
Taxonomy of Viruses, has been verified to occur in family settings $[2,6]$. Children who are infected with SARS-CoV-2 tend to have a good prognosis [5], whereas adult patients with underlying medical disorders appear to be more susceptible to significant illness associated with the virus $[1,2]$.

Pediatric patients with kidney failure (chronic kidney disease (CKD) stage 5) who are supported by maintenance incenter hemodialysis (HD) or home peritoneal dialysis (PD) are at significant risk for experiencing infectious diseases such as COVID-19 because of their compromised immune system and their frequent exposure to the hospital setting. Therefore, we have proposed a set of recommendations for

Department of Pediatrics, Tongji Hospital, Tongji Medical College of Huazhong University of Science \& Technology, Wuhan, China

5 Department of Pediatrics, Peking University First Hospital, Beijing, China

6 Department of Nephrology, Beijing Children's Hospital, Capital Medical University, Beijing, China

7 Department of Pediatrics, Yong Loo Lin School of Medicine, National University of Singapore, Singapore, Singapore

8 Division of Pediatric Nephrology, Children's Mercy Kansas City, Kansas City, MO, USA 
the prevention and control of SARS-CoV-2 infection and COVID-19 in pediatric HD centers and in home PD settings for children who reside in geographic regions where COVID19 is highly prevalent. The recommendations are based on the epidemiological features of the virus and COVID-19 disease, susceptibility factors, and preventive and control strategies. No doubt, additional recommendations for the dialysis population from agencies such as the Centers for Disease Control and Prevention in the USA will be forthcoming as well.

\section{Epidemiological features}

All age groups are susceptible to the SARS-CoV-2 infection, while the risk is increased in children because of their respiratory tract characteristics, their immature immune system, and their susceptibility to respiratory virus infections. The main infectious sources are not only patients infected by SARS-CoV-2 with clinical symptoms but also infected individuals without clinical symptoms as well $[1,4,6]$. Evidence has clearly shown that patients may transmit the virus during the viral incubation period when clinical symptoms are absent. The SARS-CoV-2 is primarily spread through respiratory droplets from an infected individual in close contact. Case-based evidence suggests the existence of aerosol and fecal-oral transmission [7]. Whether transmission can occur through mother-infant vertically or through breastfeeding has not yet been determined. Some studies are also focusing on the presence of SARS-CoV-2 in the urine of infected patients. Previous studies conducted during SARS2003 showed that the SARSrelated coronavirus could not be identified by polymerase chain reaction (PCR) or viral culture in the PD effluent of SARS patients who received PD, but that the stool PCR remained positive for 5 weeks [8]. Whether or not SARS-CoV-2 is present in the PD effluent of patients has not yet been reported. Table 1 shows the diagnostic classifications of COVID-19, including suspected cases and confirmed cases.

\section{Susceptibility factors for children with CKD stage 5 on maintenance HD or PD}

It is well recognized that people with chronic medical disorders are more likely to develop severe disease and infection is the second most common cause of death in patients with CKD stage 5. Dialysis patients with underlying lung disease and those on immunosuppressive medications are at an even higher risk for infection-related complications. An important article recently published in the Journal of the American Medical Association (JAMA) showed that in an experience from Wuhan, China, hospital-related transmission of SARSCoV-2 was suspected in $41.3 \%$ of infected patients with COVID-19, indicative of the potential risk of hospitalization to patient outcome [9]. In turn, the factors associated with an increased risk for contracting SARS-CoV-2 infection among pediatric chronic dialysis patients, especially those who receive in-center HD, include the following: (a) compromised immune system (the result of long-term malnutrition, uremia, and/or immunosuppressants); (b) close proximity to other
Table 1 Diagnostic classifications of COVID-19 (based on data from [7])

\section{Epidemiological history}

Clinical manifestations

Suspected case

Confirmed case a. A history of travel or residence in areas with a wide spread diffusion of COVID-19 within 14 days prior to the onset of symptoms.

b. Direct contact with SARS-CoV-2-infected individual (positive nucleic acid test) within 14 days before the onset of symptoms.

c. Direct contact with a patient who has symptoms of fever and/or respiratory infection from areas with a wide spread diffusion of COVID-19 within 14 days prior to the onset of symptoms.

d. Cluster onset.

a. Fever and/or respiratory symptoms.

b. Pulmonary imaging with signs of COVID-19.

c. Laboratory tests suggesting early stage of disease: total white blood cell count normal or decreased with decreased lymphocyte count.

Individual who meets one of the epidemiological history criteria combined with two of the clinical manifestations, or who meets all three of the clinical manifestations.

Suspected case with RT-PCR positive detection of SARS-CoV-2 nucleic acid, or highly homologous viral gene sequencing to the known SARS-Cov-2. 
patients during treatment in a confined HD unit; (c) frequent contact with healthcare workers, who may be asymptomatic but infected while caring for a variety of other patients; (d) a need for the presence of parents or other relatives during the treatment, which increases the risk of cluster infection; and (e) non-adherence to, or a break in, implementation of recommended infection prevention practices.

\section{Preventive and control strategies for in-center HD}

\section{Staff management (Table 2)}

Healthcare staff training Since information regarding COVID-19 is continually being updated, healthcare staff should be educated with the latest knowledge of the disease on a regular basis.

Epidemic history surveillance Any suspected epidemic history that involves a healthcare staff member should be reported to the dialysis center leadership and infectious disease experts, as defined by the particular program. The staff member should be subjected to a self-imposed quarantine for a period of at least 14 days [7].

Body temperature monitoring The body temperature of all the healthcare staff should be monitored twice daily as a surveillance activity. Any staff identified to have a high temperature (> $37.3^{\circ} \mathrm{C}$ for two consecutive measurements) or a suspected respiratory tract infection should remain at home and be closely observed. Family members and dialysis leadership should closely monitor the status of staff who remain at home for observation.
Personal protective equipment [10] Personal protective equipment (PPE) consists of a highly efficient mask, cap, gown, gloves, and goggles or face shield, which should be worn in a proper and constant manner. Within the general dialysis floor where most patients are receiving dialysis, healthcare staff should at least wear a disposable surgical or N95 mask and a disposable cap. When performing procedures that require aseptic technique, healthcare staff should wear disposable gloves. In centers with a high-risk population, it is recommended that dialysis nurses wear a gown and goggles throughout the dialysis session. When providing HD for subjects who are being observed for the presence of the virus and possible COVID-19 but no definitive diagnosis has been made, PPE should be worn according to the second level of protection standard (including N95 mask, cap, protective gown, goggles or face shield, shoe covers, and gloves). When providing HD for suspected or confirmed cases in an isolation room, PPE should be used according to the third level of protection standard (including N95 mask, cap, protective gown, powered air purifying respirator, shoe covers, and gloves). PPE is the most frequently required but also most frequently ignored aspect of infection control measures. Therefore, administrative, environmental, and engineering measures should be implemented concurrently to enhance PPE compliance.

Hand hygiene According to the "Five Moments for Hand Hygiene" recommendations from WHO [11], hand hygiene should be performed: $(1,2)$ before and after every patient contact, (3) after body fluid exposure or risk, (4) after touching a patient's immediate environment, and (5) before clean/ aseptic procedures. Hand hygiene should be performed using soap and water when hands are visibly soiled with contaminants, and if not, using alcohol-based hand rub sanitizer for
Table 2 SARS-CoV-2 infection prophylactic strategies for HD healthcare staff
Healthcare staff training

Epidemic history surveillance

Body temperature monitor

Personal protective equipment [10]
Hand hygiene

Adequate rest
Regularly provided updated information about COVID-19

Any close contact with suspected/confirmed cases or epidemic area should be reported

Body temperature and any symptoms should be monitored

Disposable surgical or N95 masks and caps are required.

HD for medical observation cases: PPE performed according to the second level of protection standard, including N95 mask, cap, protective gown, goggles or face shield, shoe covers, and gloves.

HD for suspected or confirmed cases: PPE performed according to the third level of protection standard, including N95 mask, cap, protective gown, powered air purifying respirator, shoe covers, and gloves.

Performed in accordance with the "Five Moments for Hand Hygiene" as recommended by the WHO

Adequate rest should be guaranteed 
20-30 s. Hand hygiene should also be performed even when wearing gloves for procedures. Hand hygiene is the most critical infection prevention measure. The dialysis unit should provide continuous education and supervision regarding the importance and recommended approach to hand hygiene, and provide the necessary equipment such as a sufficient number of sinks with soap dispensers, paper towels, hand lotions, and alcohol-based hand rubs placed at each patient station.

Adequate rest Healthcare staff should receive adequate rest as they need to concentrate fully at work to avoid accidental contamination. Staffing should also be appropriate to the patient volume so that the dialysis staff are not required to multitask, another risk factor for accidental contamination. The hospital and dialysis leadership should address and continuously monitor both the mental and physical health of the staff.

\section{HD patient management (Table 3)}

Patient education Basic knowledge about COVID-19 and possible precautionary measures should be shared with dialysis patients and families. Advice on proper hygiene, nutrition, and mental support should also be given.

Remote medical care Communicating with each patient and their families by telephone or Internet is recommended. Any signs of illness experienced by the HD patient or his/her family member should be reported to the dialysis program promptly. If the family member of the child has a history of epidemic exposure, he/she should be isolated from the child immediately. It is highly recommended that the patient should limit any time at the hospital other than the exact time required for dialysis. If the patient is found to have fever and/or respiratory symptoms with a history of epidemic exposure, he/she should be referred immediately to a designated fever clinic or other hospital specified location for evaluation.
Epidemic history surveillance A detailed epidemic history of both the patient and close contacts should be obtained repeatedly.

Body temperature monitoring The patients and their accompanying persons should undergo body temperature screening, as well as screening for respiratory symptoms. The screening should be conducted outside the dialysis center. If one is found to have fever and/or respiratory symptoms in association with a history of epidemic exposure, he/she should be referred to a designated fever clinic or other specified location in the hospital for evaluation.

Personal protective equipment The HD patient should wear a disposable general medical mask or surgical mask when undergoing HD. Use of an N95 mask is not recommended for HD patients since it could result in hypoxemia [12].

Limiting accompanying persons Unlike adults, young children often require caregivers to accompany them during the HD treatment and this increases the potential risk of cluster transmission. One patient should be accompanied by only one fixed caregiver, who needs to wait in the waiting area wearing a disposable general medical mask or a surgical mask. The accompanying person should remain in the waiting area throughout the dialysis session and should only be allowed to enter into the dialysis area in special situations when approved by dialysis staff. If the accompanying person is substituted by another person, the contact history of the new individual should be acquired in detail.

Inter-center transfer avoidance Except in urgent situations, transferring patients to different dialysis units should be avoided. In principle, patients with CKD stage 5 should always receive HD treatment in the same dialysis unit where they are registered. If there are not enough regular staff in the
Table 3 SARS-CoV-2 infection prophylactic strategies for HD patients

\begin{tabular}{ll}
\hline Patient education & $\begin{array}{c}\text { Provide basic knowledge of COVID-19 and repeated } \\
\text { education of recommended infection prevention practices } \\
\text { Medical consultations by phone or on-line to be encouraged }\end{array}$ \\
$\begin{array}{l}\text { Remote medical care } \\
\text { Epidemic history surveillance }\end{array}$ & $\begin{array}{c}\text { Any close contact with suspected/confirmed cases or with } \\
\text { history of presence in epidemic area should be reported; } \\
\text { this includes patients and companions }\end{array}$ \\
Body temperature monitor & $\begin{array}{l}\text { Body temperature and any symptoms should be monitored } \\
\text { both for patients and companions }\end{array}$ \\
Personal protective equipment & $\begin{array}{l}\text { Patients should be instructed to wear a disposable general } \\
\text { medical mask or a surgical mask during the HD treatment }\end{array}$ \\
Limiting accompanying persons & $\begin{array}{c}\text { One child should be accompanied by only one caregiver, who } \\
\text { would be allowed to enter into dialysis area only in special situations. }\end{array}$ \\
Inter-center transfer avoidance & Keeping the same accompanying person is recommended. \\
\hline
\end{tabular}


center, volunteers from the national pediatric nephrology society and related organizations are to be encouraged to provide assistance.

\section{HD facility management (Table 4)}

Increased disinfection frequency [13] Disinfection measures should be performed between each shift of dialysis and after all patients' treatments.

Disinfection of all the items and floor After dialysis, a 500$\mathrm{mg} / \mathrm{L}$ chlorine-containing disinfectant should be utilized to thoroughly wipe and disinfect the dialysis machine, first disinfecting the clean surface and then the contaminating surface for more than $30 \mathrm{~min}$. If any surface is contaminated by blood or any other secretions, a 2000-mg/L chlorinecontaining disinfectant should be used for more than $30 \mathrm{~min}$ after cleaning.

Disinfection of the air The room should be disinfected by ultraviolet light $\left(\geq 1.5 \mathrm{~W} / \mathrm{m}^{3}\right)$ for more than $30 \mathrm{~min}$ [14].

Fixed dialysis machine Each child should use the same dialysis machine each time HD is provided.

Proper design of HD unit All HD patients should be kept relatively isolated from one another during their treatments by spacing the dialysis chairs/beds at least $1 \mathrm{~m}$ apart. If these conditions cannot be met, curtains should be drawn around the patient during the HD session. Unnecessary talking or eating is to be avoided during dialysis.

\section{Control strategies}

Suspected/confirmed cases Children with CKD stage 5, if identified as suspected/confirmed cases, should be referred to a designated treatment hospital for COVID19 and dialysis. Severe suspected/confirmed cases requiring life support due to multiple organ dysfunction syndrome may be treated with bedside continuous kidney replacement therapy (CKRT). Children should wear disposable surgical masks while dialysis is being provided. Healthcare staff should be protected according to the third-level protection requirements [10]. Disposable items and drugs (including pipes, filters, normal saline, replacement fluid) that are in the isolation ward should not be taken out of the isolation ward. Children identified as suspected infection cases may be treated as a normal, non-infected dialysis patient after obtaining negative results for SARS-CoV-2 nucleic acid on two consecutive occasions at an interval of $24 \mathrm{~h}$.

Medical observation cases [7, 15] When there are suspected/ confirmed cases with COVID-19 in the pediatric HD center, close contacts or persons with suspected exposure, including other children on HD and medical staff, need to undergo close medical observation. The observation period is 14 days after the last contact with ineffective protection or suspected exposure with the case. If COVID-19 diagnosis is excluded for the suspected cases, close contacts or persons can be discharged from medical observation.

Since children who receive maintenance HD are required to go to the HD center 3-5 times a week for dialysis treatment, and the number of maintenance HD patients in each center is typically small, it is recommended that the pediatric HD patient under medical observation be quarantined and dialyzed in an isolated observation room or isolated dialysis area [15]. If this cannot be arranged, he/she should receive dialysis during the last dialysis session of the day when potential contact with other patients is limited. During dialysis, children should wear disposable surgical masks. Healthcare staff should be protected according to the second-level protection requirements [10]. Individuals under medical observation should be monitored for the development of any suspicious symptoms. If the child has suspicious symptoms, the relevant examination and investigations together with a multidisciplinary team consultation should be carried out. Discharge from medical observation and transfer to the general dialysis area for treatment can
Table 4 SARS-CoV-2 infection prophylactic strategies for HD facility

\begin{tabular}{ll}
\hline Increased disinfect frequency & $\begin{array}{c}\text { Disinfection measures should be performed between } \\
\text { each shift of dialysis and after all patient treatments } \\
\text { Recommended concentrations of chlorine-containing } \\
\text { disinfectant should be utilized for } 30 \text { min }\end{array}$ \\
Disinfection of the air & $\begin{array}{l}\text { Disinfection by ultraviolet light should be conducted } \\
\text { for more than } 30 \text { min }\end{array}$ \\
Fixed dialysis machine & $\begin{array}{l}\text { If possible, one child should use the same dialysis machine } \\
\text { at every treatment }\end{array}$ \\
Proper design of HD unit layout & $\begin{array}{l}\text { The separation between two HD beds/chairs should } \\
\text { be at least } 1 \mathrm{~m} \text {; curtains should be drawn around } \\
\text { patient during HD treatments. }\end{array}$ \\
\hline
\end{tabular}


occur if she/he has normal body temperature without clinical manifestations of infection with SARS-CoV-2 14 days after exposure to the contact.

\section{Preventive and control strategies for home PD (Table 5)}

Compared with in-center maintenance HD, chronic PD is the most frequently used pediatric dialysis modality worldwide. In the midst of the COVID-19 epidemic, pediatric PD patients and their families should actively strengthen various protective measures.

\section{Home environment management}

Air out the room in which PD is being conducted by opening windows and doors as deemed feasible at least twice a day for 30 min each time. When connecting the PD catheter to the dialysis tubing to conduct an exchange, it is necessary to close the window and/or any air conditioner vents to avoid convective air flow. The floor of the PD treatment room should be swept and cleansed before the PD treatment is conducted once daily, followed by ultraviolet disinfection $\left(\geq 1.5 \mathrm{~W} / \mathrm{m}^{3}\right)$ of the room's air, if ultraviolet equipment is available at home. The ultraviolet disinfection period should be no less than $30 \mathrm{~min}$ each time [13]. The ultraviolet lamp should be kept clean and wiped with $75 \%$ alcohol once a week. Before and after each manual PD exchange, the procedure table should be wiped with a $75 \%$ alcohol wet cloth. If using an automated PD (APD) machine, a 75\% alcohol wet cloth should be used to wipe the APD machine before and after each treatment.

\section{Patient management}

Patient/family education Home PD patients and their families should be educated on the SARS-CoV-2 infection prevention strategies; it is recommended that both children and caregivers try to stay home and avoid crowded places. If going out, they should wear a mask and avoid touching surfaces unnecessarily.
Hand hygiene Patients and care providers should wash hands in accordance with the seven-step washing method. Hand hygiene should be performed (a) after using the rest room and before meals; (b) upon returning home; (c) after contacting garbage or touching animals; (d) before and after the PDrelated procedures; and (e) after the disposal of the PD drainage fluid. It is necessary to wash hands with soap and hand sanitizer before conducting PD. Antibacterial hand soap is recommended.

Protective equipment During the home PD procedure, the care provider/operator should wear a general medical mask and cap, and the child should wear a general medical mask. The mask should cover the nose and mouth, and the cap should cover the hair.

Disinfection of drainage fluid The PD effluent should be disinfected for all PD patients in regions where COVID-19 is highly prevalent. It is recommended that the PD effluent be mixed with $500 \mathrm{mg} / \mathrm{L}$ chlorine-containing solution for $1 \mathrm{~h}$ and poured into the toilet.

Online medical consultation for home PD Online medical consultation is recommended for home PD patients so as to reduce unnecessary hospital visits for children and caregivers during the epidemic. It is also recommended that, if available, a remote management system with remote connectivity should be used to facilitate effective monitoring of home $\mathrm{PD}$, and as a means to provide timely feedback and improve treatment compliance and efficacy.

\section{Conclusion}

Children on chronic dialysis are particularly susceptible to COVID-19. In order to effectively prevent and control the transmission of SARS-CoV-2 among children who receive maintenance dialysis, we formulated this set of recommendations based on infectious disease guidelines and our experience with the COVID-19 epidemic, which healthcare staff in pediatric dialysis centers can refer to. We suspect that these
Table 5 SARS-CoV-2 infection prophylactic strategies for home PD patients

\begin{tabular}{|c|c|}
\hline \multirow{3}{*}{$\begin{array}{l}\text { Home environment } \\
\text { management }\end{array}$} & Air the room at least twice a day for $30 \mathrm{~min}$ each time. \\
\hline & $\begin{array}{l}\text { Sweep and clean the floor before the PD treatment once a day, and then carry out } \\
\text { ultraviolet disinfection. }\end{array}$ \\
\hline & $\begin{array}{l}\text { Before and after each PD treatment, use } 75 \% \text { alcohol wet cloth to wipe the procedure } \\
\text { table or APD machine (as appropriate for patient). }\end{array}$ \\
\hline \multirow[t]{4}{*}{ Patient management } & Learn the SARS-CoV-2 infection prevention information. \\
\hline & Strictly perform hand hygiene and PPE. \\
\hline & Disinfection of drainage fluid with $500 \mathrm{mg} / \mathrm{L}$ chlorine-containing solution for $1 \mathrm{~h}$. \\
\hline & Online medical consultation for home PD. \\
\hline
\end{tabular}


recommendations may well apply during epidemics related to other respiratory viruses as well. These recommendations will be updated as new information regarding SARS-CoV-2 and COVID-19 becomes available.

Acknowledgments The authors thank Dr. Chuanqing Wang from Department of Nosocomial Infection Control, Children's Hospital of Fudan University and Dr. Xiaorong Xiang from Department of Nosocomial Infection Control, Children's Hospital of Nanjing Medical University for their recommendation of nosocomial infection control.

\section{Compliance with ethical standards}

Conflict of interest The authors declare that they have no competing interests.

\section{References}

1. Zhu N, Zhang D, Wang W, Li X, Yang B, Song J, Zhao X, Huang B, Shi W, Lu R, Niu P, Zhan F, Ma X, Wang D, Xu W, Wu G, Gao GF, Tan W (2020) A novel coronavirus from patients with pneumonia in China, 2019. N Engl J Med 382:727-733

2. Huang C, Wang Y, Li X, Ren L, Zhao J, Hu Y, Zhang L, Fan G, Xu J, Gu X, Cheng Z, Yu T, Xia J, Wei Y, Wu W, Xie X, Yin W, Li H, Liu M, Xiao Y, Gao H, Guo L, Xie J, Wang G, Jiang R, Gao Z, Jin Q, Wang J, Cao B (2020) Clinical features of patients infected with 2019 novel coronavirus in Wuhan, China. Lancet 395:497-506

3. Johns Hopkins University (Accessed 23 March, 2020). Coronavirus Resource Center. https://coronavirus.jhu.edu/map. html

4. Novel Coronavirus Pneumonia Emergency Response Epidemiology Team (2020) The epidemiological characteristics of an outbreak of 2019 novel coronavirus diseases (COVID-19) in China. Zhonghua Liu Xing Bing Xue Za Zhi 41:145-151

5. Lu X, Zhang L, Du H, Zhang J, Li YY, Qu J, Zhang W, Wang Y, Bao S, Li Y, Wu C, Liu H, Liu D, Shao J, Peng X, Yang Y, Liu Z, Xiang Y, Zhang F, Silva RM, Pinkerton KE, Shen K, Xiao H, Xu S, Wong GWK, Chinese Pediatric Novel Coronavirus Study Team (2020) SARS-CoV-2 infection in children. N Engl J Med. https:// doi.org/10.1056/NEJMc2005073

6. Chan JF, Yuan S, Kok KH, To KK, Chu H, Yang J, Xing F, Liu J, Yip CC, Poon RW, Tsoi HW, Lo SK, Chan KH, Poon VK, Chan
WM, Ip JD, Cai JP, Cheng VC, Chen H, Hui CK, Yuen KY (2020) A familial cluster of pneumonia associated with the 2019 novel coronavirus indicating person-to-person transmission: a study of a family cluster. Lancet 395:514-523

7. National Health Commission of China (2020) New coronavirus pneumonia prevention and control program (7th edition). http:// ww w.nhc.gov.cn/x c s/zheng cwj/202003/ 46 c 9294 a 7 d fe 4 cef 80 d c 7 f 5912 eb $1989 /$ files/ ce3e6945832a438eaae415350a8ce964.pdf

8. Kwan BC, Leung CB, Szeto CC, Wong VW, Cheng YL, Yu AW, Li PK (2004) Severe acute respiratory syndrome in dialysis patients. J Am Soc Nephrol 15:1883-1888

9. Wang D, Hu B, Hu C, Zhu F, Liu X, Zhang J, Wang B, Xiang H, Cheng Z, Xiong Y, Zhao Y, Li Y, Wang X, Peng Z (2020) Clinical characteristics of 138 hospitalized patients with 2019 novel coronavirus-infected pneumonia in Wuhan, China. JAMA. https:// doi.org/10.1001/jama.2020.1585

10. National Health Commission of China (2016) Regulation for prevention and control of healthcare associated infection of airborne transmission disease in healthcare facilities. http://www.nhc.gov.cn/ ewebeditor/uploadfile/2017/01/20170119150530360.pdf

11. World Health Organization (2009) WHO guidelines on hand hygiene in health care: first global patient safety challenge clean care is safer care. https://apps.who.int/iris/bitstream/handle/10665/44102/ 9789241597906 eng.pdf

12. Kao TW, Huang KC, Huang YL, Tsai TJ, Hsieh BS, Wu MS (2004) The physiological impact of wearing an N95 mask during hemodialysis as a precaution against SARS in patients with end-stage renal disease. J Formos Med Assoc 103:624-628

13. Wang M, Beijing Hemodialysis Quality Control and Improvement Center, First Hospital, Peking University, Beijing 100034 China (2004) The incidence and prevention of severe acute respiratory syndrome in 81 hemodialysis units in Beijing. Zhonghua Nei Ke Za Zhi 43:420-422

14. National Health Commission of China (2012) Management specification of air cleaning technique in hospitals. http://www.nhc.gov. $\mathrm{c} \mathrm{n} / \mathrm{wjw} / \mathrm{s} 9496 / 201204 / 54511 / \mathrm{files} /$ 8df30d0236d3421c87492786c55c26e7.pdf

15. Park HC, Lee YK, Lee SH, Yoo KD, Jeon HJ, Ryu DR, Kim SN, Sohn SH, Chun RW, Choi KB (2017) Middle East respiratory syndrome clinical practice guideline for hemodialysis facilities. Kidney Res Clin Pract 36:111-116

Publisher's note Springer Nature remains neutral with regard to jurisdictional claims in published maps and institutional affiliations. 